УДК 538.915

DOI: https://doi.org/10.17308/kcmf.2019.21/756

Поступила в редакцию 17.04.2019

Подписана в печать 15.05.2019

\title{
ВЛИЯНИЕ ПЕРЕХОДНЫХ МЕТАЛЛОВ ІІІВ-ГРУППЫ НА ФОРМИРОВАНИЕ ЗАМКНУТЫХ ГЕРМАНИЕВЫХ КЛАСТЕРОВ: КОМПЬЮТЕРНЫЙ ЭКСПЕРИМЕНТ В РАМКАХ ТЕОРИИ ФУНКЦИОНАЛА ПЛОТНОСТИ
}

\author{
(2019 Н. А. Борщ ${ }^{1 \bowtie}$, С. И. Курганский ${ }^{2}$ \\ ${ }^{1}$ Воронежский государственный технический университет \\ Московский пр., 14, 394026 Воронеж, Российская Федерация \\ ${ }^{2}$ Воронежский государственный университет \\ Университетская пл., 1, 394018 Воронеж, Российская Федерация
}

\begin{abstract}
Аннотация. Представлены результаты моделирования пространственной структуры и электронных свойств кластеров $\mathrm{MeGe}_{16}^{-}$и $\mathrm{MeGe}_{20}^{-}(\mathrm{Me}=\mathrm{Sc}, \mathrm{Y}, \mathrm{Lu})$. Рассматривается возможность синтеза фуллереноподобных кластеров и кластеров с другими типами замкнутых структур. Проведены сравнительные расчеты в рамках теории функционала плотности с использованием базиса SDD и трех различных потенциалов - B3LYP, B3PW91 и PBEPBE. Анализируется влияние выбора потенциала на результаты моделирования пространственной структуры кластеров и их электронного спектра. Оценка адекватности теоретических методов проводится путем сравнения рассчитанных электронных спектров с экспериментальными результатами по фотоэлектронной спектроскопии кластеров.
\end{abstract}

Ключевые слова: атомные кластеры, электронная структура, фуллерены, теория функционала плотности, фотоэлектронные спектры.

\section{ВВЕДЕНИЕ}

Основной тенденцией в современной физике конденсированных сред, химии, материаловедении является поиск новых функциональных материалов, которые могли бы применяться для развития как нанотехнологий, так и других технологий будущего. Это должны быть материалы с принципиально новыми свойствами, а значит, и с принципиально новой атомной структурой и размерностью. Многие современные исследования посвящены изучению и модификации свойств фуллеренов, графенов и других структур на основе углерода. Но еще в 1985 году, после синтеза фуллерена [1], началась работа по поиску аналогичных структур, формируемыми другими элементами IV группы - кремнием, германием, оловом [2]. С тех пор было установлено, что эти элементы также могут формировать как фуллереноподобные структуры, так и другие виды замкнутых структур [3-9].

Большинство этих структур стабилизируется атомом переходного металла, сорт которого мо-

$\triangle$ Борщ Надежда Алексеевна,

e-mail: borsch-nadya@yandex.ru жет существенно влиять на их геометрическую структуру и электронные свойства [3-14]. Такие инкапсулированные кластеры служат в свою очередь элементами для конструирования структур более высокой размерности, в том числе наноклеточных кристаллов, которые также называют клатратами [15]. Их элементарная ячейка формируется из фуллереноподобных кластеров кремния, германия, олова, инкапсулирующих атом металла. Оказалось, что такие свойства, как электропроводность, теплопроводность и некоторые механические характеристики зависят от сорта атома металла [16-17].

Очевидно, что для синтеза новых материалов необходимо знать особенности пространственной структуры кластеров, механизмы их формирования, а также электронные свойства и закономерности их зависимости от размера, геометрии и атомного состава кластера. Экспериментальные методы пока ограничены в своих возможностях и могут дать ответы далеко не на все вопросы, поэтому на первое место выходят методы компьютерного эксперимента. В свою очередь, многочисленные расчеты показали, что результаты компьютерного моделирования мо- 
гут сильно зависеть от используемого метода и кардинально отличаться для одних и тех же систем [7, 18]. Поэтому оптимальным методом исследования нольмерных объектов в настоящее время является комбинирование экспериментальных и теоретических методов.

В данной работе представлены результаты моделирования пространственной структуры и электронных свойств кластеров $\mathrm{MeGe}_{16}^{-}$и $\mathrm{MeGe}_{20}^{-}$ $(\mathrm{Me}=\mathrm{Sc}, \mathrm{Y}, \mathrm{Lu})$. Рассматривается возможность синтеза фуллереноподобных кластеров и кластеров с другими типами замкнутых структур. Проведены сравнительные расчеты в рамках теории функционала плотности с использованием базиса SDD [19] и трех различных потенциалов - B3LYP [20, 21], B3PW91 [22, 23] и PBEPBE [24]. Анализируется влияние выбора потенциала на результаты моделирования пространственной структуры кластеров и их электронного спектра. Оценка адекватности теоретических методов проводится путем сравнения рассчитанных электронных спектров с экспериментальными результатами по фотоэлектронной спектроскопии кластеров [25].

\section{МЕТОД РАСЧЕТА}

В результате расчета электронного спектра получались собственные значения энергии каждой молекулярной орбитали, т. е. энергетический спектр, в котором каждую молекулярную орбиталь можно представить в виде уровня. Теоретические электронные спектры получались после того, как каждый энергетический уровень заменялся гауссовым распределением с шириной $0.15 \mathrm{eV}$, и интенсивности всех распределений при каждом значении энергии складывались.

Средняя энергия связи $E_{b}$ вычислялась по формуле:

$$
E_{b}=\frac{n E(\mathrm{Ge})+E\left(\mathrm{Me}^{-}\right)-E\left(\mathrm{MeGe}_{n}^{-}\right)}{n+1},
$$

где $E(\mathrm{Ge})$ и $E\left(\mathrm{Me}^{-}\right)$- полные энергии свободных атома германия и аниона металла, соответственно, $E\left(\mathrm{MeGe}_{n}^{-}\right)$- полная энергия кластера, $n$ - число атомов германия в кластере.

\section{РЕЗУЛЬТАТЫ И ИХ ОБСУЖДЕНИЕ}

На рис. 1 показаны структуры наиболее стабильных изомеров кластеров $\mathrm{MeGe}_{16}^{-}$и $\mathrm{MeGe}_{20}^{-}$ $(\mathrm{Me}=\mathrm{Sc}, \mathrm{Y}, \mathrm{Lu})$, на рис. 2 - сопоставление рассчитанных плотностей электронных состояний с фотоэлектронными спектрами [25], а в табл. 1 приведены разности между средними энергиями связи в основных и побочных изомерах. Профили плотности электронных состояний практически не зависят от потенциала, использованного в расчете, и зависят только от структуры изомера, поэтому на рисунке показаны только рассчитанные электронные спектры, полученные в расчете с использованием потенциала B3PW91.

Основной изомер всех трех кластеров $\mathrm{MeGe}_{16}^{-}$ $(\mathrm{Me}=\mathrm{Sc}, \mathrm{Y}, \mathrm{Lu})$ имеет структуру многогранника с треугольными гранями, так называемого многогранника Франка-Каспера (изомер 16-FK). Побочные изомеры также имеют эндоэдральную

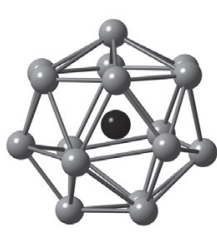

16-FK

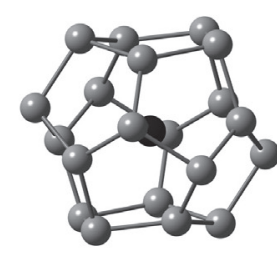

20-full

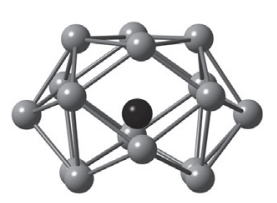

16-ph

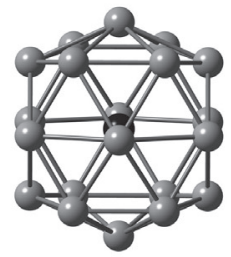

20-FK

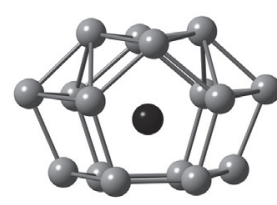

16-ph2

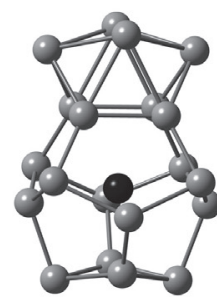

20-full 16

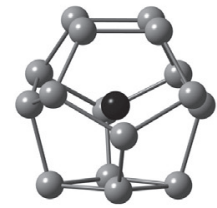

16-full

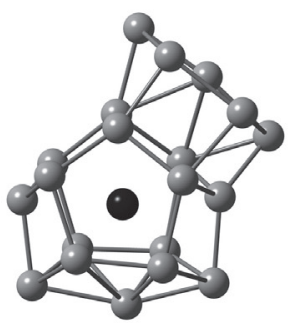

20-pr

Рис. 1. Пространственная структура наиболее стабильных изомеров кластеров $\mathrm{MeGe}_{16}^{-}$и $\mathrm{MeGe}_{20}^{-}$ $(\mathrm{Me}=\mathrm{Sc}, \mathrm{Y}, \mathrm{Lu})$

[Fig. 1. Spatial structure of stable isomers of $\mathrm{MeGe}_{16}^{-}$and $\mathrm{MeGe}_{20}^{-}(\mathrm{Me}=\mathrm{Sc}, \mathrm{Y}, \mathrm{Lu})$ clusters] 

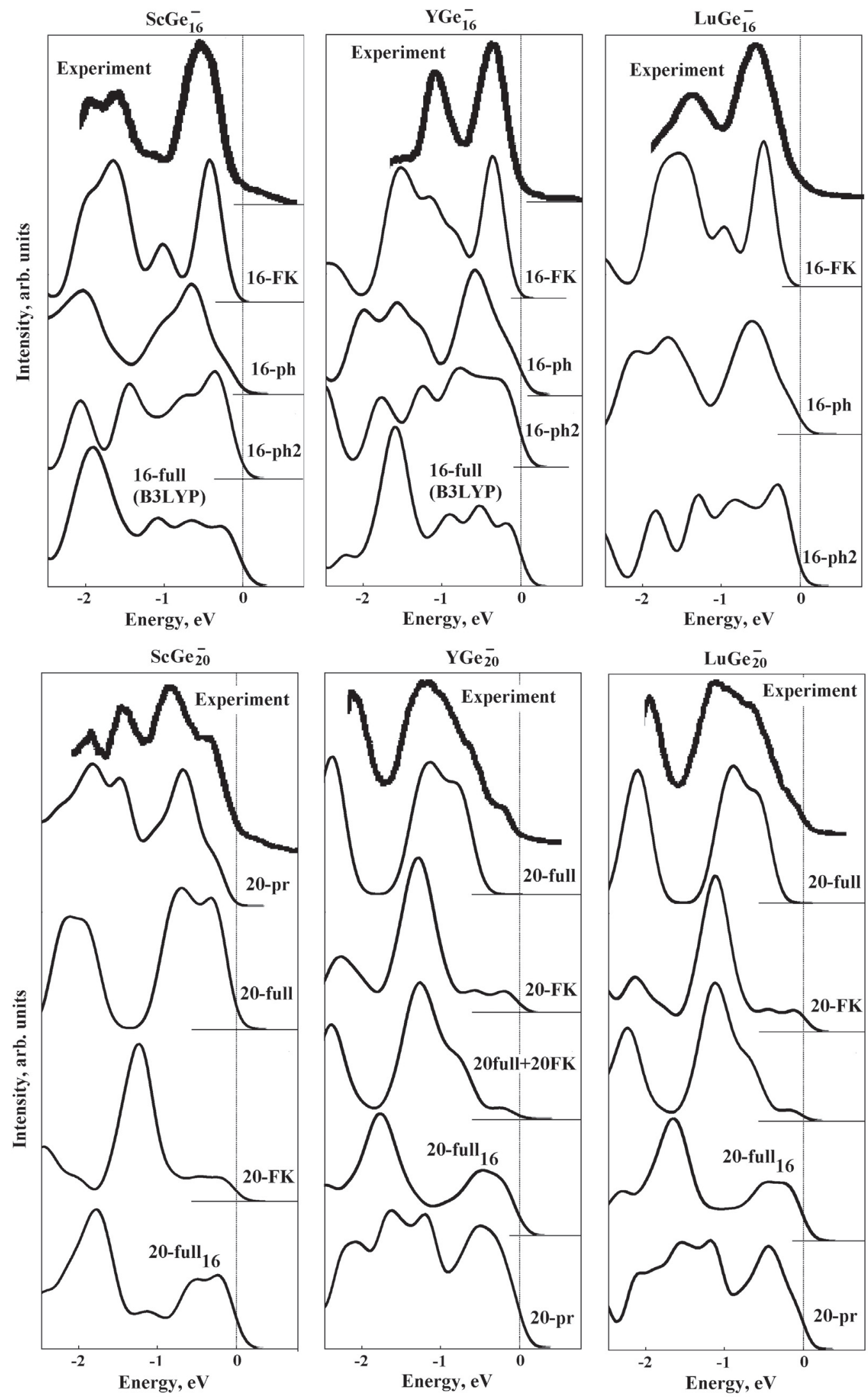

Рис. 2. Сравнение рассчитанных электронных спектров кластеров $\mathrm{MeGe}_{16}^{-}$и $\mathrm{MeGe}_{20}^{-}(\mathrm{Me}=\mathrm{Sc}, \mathrm{Y}, \mathrm{Lu})$ с экспериментальными фотоэлектронными [25]

[Fig. 2. Comparison of the calculated electron spectra of $\mathrm{MeGe}_{20}^{-}$and $\mathrm{MeGe}_{20}^{-}$clusters and $(\mathrm{Me}=\mathrm{Sc}, \mathrm{Y}, \mathrm{Lu})$ with experimental photoelectron spectra [25]] 
Таблица 1. Разность между средними энергиями связи $\Delta \mathrm{E}_{\mathrm{b}}$ (эВ/атом) основного и побочных изомеров кластеров $\mathrm{MeGe}_{16}^{-}$и $\mathrm{MeGe}_{20}^{-}(\mathrm{Me}=\mathrm{Sc}, \mathrm{Y}, \mathrm{Lu})$ при расчете в разных потенциалах

[Table 1. The difference between the average binding energies $\Delta \mathrm{E}_{\mathrm{b}}(\mathrm{eV} / \mathrm{atom})$ of the main and minor isomers of the $\mathrm{MeGe}_{16}^{-}$and $\mathrm{MeGe}_{20}^{-}$clusters $(\mathrm{Me}=\mathrm{Sc}, \mathrm{Y}, \mathrm{Lu}$ ) in calculations with different potentials]

\begin{tabular}{|c|c|c|c|}
\hline \multirow{2}{*}{ Кластер } & \multicolumn{3}{|c|}{$\Delta E_{\mathrm{b}}$} \\
\cline { 2 - 4 } [Cluster] & B3PW91 & B3LYP & PBEPBE \\
\hline Sc-16-FK & 0.0000 & 0.0000 & 0.0000 \\
\hline Sc-16-ph & -0.0271 & -0.0154 & -0.0299 \\
\hline Sc-16-ph2 & -0.0482 & -0.0285 & -0.0532 \\
\hline Sc-16-full & - & -0.0749 & - \\
\hline Y-16-FK & 0.0000 & 0.0000 & 0.0000 \\
\hline Y-16-ph & -0.0122 & -0.0008 & -0.0153 \\
\hline Y-16-ph2 & -0.0317 & -0.0143 & -0.0349 \\
\hline Y-16-full & - & -0.0550 & - \\
\hline Lu-16-FK & 0.0000 & 0.0000 & 0.0000 \\
\hline Lu-16-ph & -0.0190 & -0.0075 & -0.0223 \\
\hline Lu-16-ph2 & -0.0390 & -0.0212 & -0.0428 \\
\hline Lu-16-full & - & - & - \\
\hline Sc-20-full & -0.0157 & -0.0011 & -0.0177 \\
\hline Sc-20-FK & -0.0326 & -0.0479 & -0.0107 \\
\hline Sc-20-full 16 & -0.0234 & -0.0141 & -0.0259 \\
\hline Sc-20-pr & 0.0000 & 0.0000 & 0.0000 \\
\hline Y-20-full & 0.0000 & 0.0000 & -0.0051 \\
\hline Y-20-FK & -0.0144 & -0.0455 & 0.0000 \\
\hline Y-20-full ${ }_{16}$ & -0.0404 & -0.0469 & -0.0461 \\
\hline Y-20-pr & -0.0434 & -0.0551 & -0.0456 \\
\hline Lu-20-full & 0.0000 & 0.0000 & -0.0100 \\
\hline Lu-20-FK & -0.0090 & -0.0409 & 0.0000 \\
\hline Lu-20-full 16 & -0.0285 & -0.0345 & -0.0377 \\
\hline Lu-20-pr & -0.0185 & -0.0310 & -0.0243 \\
\hline & & & \\
\hline
\end{tabular}

структуру - полиэдр с четырехугольными гранями (изомер 16-ph) и полиэдр с четырех- и пятиугольными гранями (изомер 16-ph2). Фуллереноподобный многогранник (изомер 16-full) при оптимизации с использованием потенциалов B3PW91 и РBEPBE трансформировался в структуру Франка-Каспера и только при оптимизации кластеров $\mathrm{ScGe}_{16}^{-}$и $\mathrm{YGe}_{16}^{-}$в потенциале B3LYP сохранил фуллереноподобную структуру, хотя и претерпел деформацию. В расчете с использованием B3LYP-потенциала средние энергии связи в изомерах 16-FK и 16-рh близки, а профили их электронных спектров идентичны и хорошо согласуются с экспериментальным фотоэлектронным спектром [25]. Расчеты в потенциалах B3PW91 и PBEPBE показывают заметно большую разницу в средних энергиях связи этих структур. Учитывая, что рассчитанный электронный спектр изомера 16-ph хорошо согласуется с экспериментальным фотоэлектронным [25], не- льзя исключить, что он, наряду с основным изомером 16-FK, также может быть детектирован в эксперименте.

Изомер 20-full был получен в результате оптимизации правильного додэкаэдра с атомами германия в вершинах и с инкапсулированным атомом металла. В процессе оптимизации додекаэдр исказился. Структура изомера 20-FK многогранник с треугольными гранями, центрированный атомом металла. Изомер 20-full 16 сформирован на основе фуллереноподобного многогранника с шестнадцатью вершинами добавлением четырех дополнительных атомов германия. Атом металла инкапсулирован в фуллереноподобном многограннике. Изомер 20-pr имеет в основе своей структуры сильно искаженную шестиугольную призму с дополнительными атомами германия.

Для кластера $\mathrm{ScGe}_{20}^{-}$основным изомером является изомер с призматической структурой 
(Sc-20-pr). Этот результат показали оптимизационные расчеты во всех трех потенциалах. Вторым по стабильности изомером, согласно B3PW91- и B3LYP-расчетам, является изомер с фуллереноподобной структурой (Sc-20-full), a coгласно РВЕРВЕ-расчету - изомер со структурой многогранника Франка-Каспера (Sc-20-FK), однако средняя энергия связи фуллереноподобного изомера в этом расчете лишь немного меньше, чем у изомера Sc-20-FK. В расчетах с использованием потенциалов B3PW91 и РВЕРВЕ изомер Sc-20-full ${ }_{16}$ является наименее стабильным из всех рассмотренных, а только B3LYP-расчет показал, что наименее стабилен изомер со структурой Франка-Каспера.

Для кластера $\mathrm{YGe}_{20}^{-}$расчеты с использованием потенциалов B3LYP и B3PW91 показывают, что основным изомером является кластер с фуллереноподобной структурой (Y-20-full), тогда как PBEPBE-расчет указывает на то, что основным является изомер со структурой многогранника Франка-Каспера (Y-20-FK). При этом разница в средних энергиях связи между фуллереноподобной структурой и структурой Франка-Каспера в B3LYP-расчете значительна и, основываясь на ней, можно заключить, что существование кластера со структурой Франка-Каспера маловероятно. Однако в РВЕРВЕ-расчете энергии этих изомеров практически равны, в B3PW91-расчете разница между их энергиями невелика, т. е. эти результаты показывают, что можно считать обе эти структуры равновероятно детектируемыми экспериментально. Рассчитанный электронный спектр фуллереноподобного изомера неплохо согласуется с экспериментальным [25], согласие с экспериментом спектра изомера со структурой Франка-Каспера значительно хуже, но профиль их суммарной плотности электронных состояний практически в точности повторяет профиль экспериментального фотоэлектронного спектра [25]. Рассчитанные спектры изомеров $20-$ full $_{16}$ и 20-pr также хорошо согласуются с экспериментальным, однако во всех расчетах эти изомеры имеют существенно меньшую среднюю энергию связи, чем первые два, поэтому их наблюдение в эксперименте маловероятно, хотя и не может быть исключено.

С большим энергетическим отрывом основным изомером кластера $\mathrm{LuGe}_{20}^{-}$в B3LYP-расчете является фуллереноподобный изомер Lu-20-full. Этот изомер также основной и в B3PW91-расчете, однако разница с средних энергиях связи у этого изомера и у изомера $\mathrm{Lu}-20-\mathrm{FK}$ невелика.

В расчете с потенциалом РВЕРВЕ основным является изомер Lu-20-FK, но фуллереноподобный изомер имеет немногим меньшую среднюю энергию связи. Сопоставление рассчитанного и экспериментального [25] электронных спектров показывает картину, аналогичную спектрам кластера $\mathrm{YGe}_{20}^{-}$: рассчитанный электронный спектр изомера Lu-20-full неплохо согласуется с экспериментальным, спектр изомера Lu-20-FK показывает плохое согласие, но профиль их суммарной плотности электронных состояний в точности повторяет профиль экспериментального фотоэлектронного спектра [25].

Таким образом, можно заключить, что основной изомер кластеров $\mathrm{MeGe}_{16}^{-}$- многогранник Франка-Каспера для всех трех инкапсулированных атомов металла. Для кластера $\mathrm{ScGe}_{20}^{-}$ изомером, детектируемым экспериментально, является изомер с призматической структурой. Эндоэдральные структуры имеют заметно меньшие средние энергии связи, но поскольку их электронные спектры неплохо согласуются с экспериментальными (хотя и хуже, чем спектры призматического изомера), их существование вероятно. Основные изомеры кластеров $\mathrm{YGe}_{20}^{-}$ и $\mathrm{LuGe}_{20}^{-}$имеют эндоэдральные структуры, которые могут равновероятно наблюдаться в эксперименте, поскольку их суммарный спектр идеально согласуется с экспериментальным [23]. Отличия в структуре основных изомеров кластеров $\mathrm{MeGe}_{20}^{-}$связаны с размером атомов скандия, иттрия и лютеция. Атом скандия имеет заметно меньший атомный радиус (1.63 $\AA$ ), чем атомы Y и Lu (1.78 и $1.72 \AA ̊$ соответственно) [25], поэтому при большом числе атомов германия в кластере $\mathrm{ScGe}_{20}^{-}$атом скандия не может стабилизировать замкнутую германиевую решетку, и формирование эндоэдральных кластеров становится практически невозможным.

Для кластеров $\mathrm{MeGe}_{16}^{-}$расчеты во всех трех потенциалах дают идентичные результаты. Для кластеров $\mathrm{MeGe}_{20}^{-}$в потенциале B3LYP отмечается смещение наибольших средних энергий связи в сторону фуллереноподобных структур, а структуры Франка-Каспера в этом расчете являются наименее стабильными. Расчет с использованием потенциала РВЕРВЕ дает другую картину, структуры Франка-Каспера и фуллереноподобные структуры имеют практически одинаковые средние энергии связи. B3PW91-расчет показывает небольшую энергетическую разницу между этими двумя типами структур. Очевидно, что при таких расхождениях в результатах, единственным критерием оценки их адекватности является эксперимент, в частности известные эк- 
спериментальные данные по фотоэлектронной спектроскопии кластеров.

\section{ЗАКЛЮЧЕНИЕ}

Для кластера $\mathrm{MeGe}_{16}^{-}(\mathrm{Me}=\mathrm{Sc}, \mathrm{Y}, \mathrm{Lu})$ основной изомер имеет высокосимметричную структуру многогранника Франка-Каспера. Для кластеров $\mathrm{YGe}_{20}^{-}$и $\mathrm{LuGe}_{20}^{-}$равновероятно существование кластеров двух типов - с фуллереноподобной структурой и со структурой многогранника Франка-Каспера. Из-за существенно меньшего атомного радиуса атома скандия наиболее стабильный изомер кластера $\mathrm{ScGe}_{20}^{-}$имеет экзоэдральную структуру, в основе которой сильно искаженная шестиугольная призма. Существование эндокластеров $\mathrm{ScGe}_{20}^{-}$маловероятно.

В расчетах с использованием B3LYP-потенциала структуры Франка-Каспера могут иметь заниженные средние энергии связи, а фуллереноподобные структуры - напротив, завышенные. Расчеты с использованием потенциалов B3PW91 и PBEPBE дают результаты, наилучшим образом согласующиеся с экспериментальными данными.

\section{БЛАГОДАРНОСТИ}

Расчеты проводились в программе Gaussian09 [26], для визуализации структур использована программа GausView [26]. Для расчетов использованы ресурсы Суперкомпьютерного центра ВГУ.

\section{КОНФЛИКТ ИНТЕРЕСОВ}

Авторы декларируют отсутствие явных и потенциальных конфликтов интересов, связанных с публикацией настоящей статьи.

\section{СПИСОК ЛИТЕРАТУРЫ}

1. Kroto H. W., Heath J. R., O’Brien S. C., Curl R. F., Smalley R. E. C60: Buckminsterfullerene // Nature, 1985, v. 318, pp. 162-163. DOI: 10.1038/318162a0

2. Hiura H., Miyazaki T., Kanayama T. Formation of Metal-Encapsulating Si Cage Clusters // Phys. Rev. Lett., 2001, v. 86, p. 1733. DOI: 10.1103/PhysRevLett.86.1733

3. Wang J., Han J. Geometries, stabilities, and electronic properties of different-sized $\mathrm{ZrSin}(\mathrm{n}=1-16)$ clusters: A density-functional investigation // J. Chem. Phys., 2005, v. 123(6), pp. 064306-064321. DOI: $10.1063 / 1.1998887$

4. Guo L.-J., Liu X., Zhao G.-F. Computational investigation of TiSin $(n=2-15)$ clusters by the densityfunctional theory // J. Chem. Phys., 2007, v. 126(23), pp. 234704-234710. DOI: 10.1063/1.2743412

5. Li J., Wang G., Yao C., Mu Y., Wan J., Han M. Structures and magnetic properties of SinMn $(n=1-15)$ clusters // J. Chem. Phys., 2009, v. 130(16), pp. 164514164522. DOI: $10.1063 / 1.3123805$

6. Борщ Н.А., Берестнев К.С., Переславцева Н. С., Курганский С. И. Пространственная структура и электронный спектр кластеров YSi-n (n=6-17) // Физика твердого тела, 2014, т. 56(6), с. 1227-1232. URL: https://journals.ioffe.ru/articles/viewP$\mathrm{DF} / 26923$

7. Borshch N., Kurganskii S. Geometric structure, electron-energy spectrum, and growth of anionic scandium-silicon clusters ScSin- $(\mathrm{n}=6-20) / / \mathrm{J}$. Appl. Phys., 2014, v. 116(12), pp. 124302-1 - 124302-8. DOI: $10.1063 / 1.4896528$

8.БорщН.А.,Переславцева Н.С.,Курганский С. И. Пространственная структура и электронный спектр кластеров TiSi (n=6-18) // Журнал физической химии, 2014, т. 88(10), с. 1523-1529. DOI: 10.7868/ S0044453714100094

9. Борщ Н.А., Переславцева Н.С., Курганский С. И. Пространственная и электронная структура германий-танталовых кластеров TaGen- $(\mathrm{n}=8-17) / /$ Физика твердого тела, 2014, т. 56(11), с. 2259-2264. URL: https://journals.ioffe.ru/articles/viewPDF/41024

10. Huang X., Yang J. Probing structure, thermochemistry, electron affinity, and magnetic moment of thulium-doped silicon clusters TmSi $n(n=3-10)$ and their anions with density functional theory // J. Mol. Model., 2018, v. 24(1), p. 29. DOI: 10.1007/s00894-0173566-7

11. Zhang, Y., Yang, J., Cheng, L. J. Probing Structure, Thermochemistry, Electron Affinity and Magnetic Moment of Erbium-Doped Silicon Clusters ErSin $(\mathrm{n}=3-10)$ and Their Anions with Density Functional Theory // Clust. Sci., 2018, v. 29(2), pp. 301-311. DOI: 10.1007/s10876-018-1336-z

12. Ye T., Luo C., Xu B., Zhang S., Song H., Li G. Probing the geometries and electronic properties of charged Zr2Si n q $(\mathrm{n}=1-12, \mathrm{q}= \pm 1)$ clusters // Struct. Chem., 2018, v. 29(1), pp. 139-146. DOI: 10.1007/ s11224-017-1011-2

13. Nguyen M. T., Tran Q. T., Tran V. T. A CASSCF/ CASPT2 investigation on electron detachments from ScSi n - $(\mathrm{n}=4-6)$ clusters // J. Mol. Model., 2017, v. 23(10), p. 282. DOI: 10.1007/s00894-017-3461-2

14. Liu Y., Jucai Yang J., Cheng L. Structural Stability and Evolution of Scandium-Doped Silicon Clusters: Evolution of Linked to Encapsulated Structures and Its Influence on the Prediction of Electron Affinities for ScSin $(\mathrm{n}=4-16)$ Clusters // Inorg. Chem., 2018, v. 57(20), pp 12934-12940. DOI: 10.1021/ acs.inorgchem.8b02159

15. Kasper J. S., Hagenmuller P., Pouchard M., Cros C. Clathrate Structure of Silicon Na8Si46 and NaxSi136 (x < 11) // Science, 1965, v. 150(3704), pp. 1713-1714. DOI: 10.1126/science.150.3704.1713

16. Борщ Н. А., Переславцева Н. С., Курганский С. И. Электронная структура и спектральные 
харакеристики Cd-замещенных германиевых клатратов // Физика и техника полупроводников, 2010, т. 44(8), с. 1021-1026.

17. Борщ Н. А., Переславцева Н. С., Курганский С. И. Электронная структура и спектральные характеристики Zn-замещенных клатратных силицидов // Физика и техника полупроводников, 2011, т. 45(6), с. 729-739.

18. Reveles J. U., Khanna S. N. Electronic counting rules for the stability of metal-silicon clusters // Phys. Rev B., 2006, v. 74(3), pp. 035435-1 - 035435-6. DOI: 10.1103/PhysRevB.74.035435

19. Dunning Jr. T. H. and Hay P. J. in Modern Theoretical Chemistry / Ed. by H. F. Schaefer III, v. 3, Plenum, New York, 1977, pp. 1-28.

20. Lee C., Yang W., Parr R.G. Development of the Colle-Salvetti correlation-energy formula into a functional of the electron density // Phys. Rev. B., 1998, v. 37(2), pp. 785-789. DOI: 10.1103/PhysRevB.37.785
21. Becke A. D. Density-functional thermochemistry. III. The role of exact exchange// J. Chem. Phys., 1993, v. 98(7), pp. 1372-1377. DOI: 10.1063/1.464913

22. Becke A. D. Density functional calculations of molecular bond energies // J. Chem. Phys., 1986, v. 84(8), pp. 4524-4529. DOI: 10.1063/1.450025

23. Becke A. D. Density-functional thermochemistry. III. The role of exact exchange // J. Chem. Phys., 1993, v. 98(7), pp. 5648-5652. DOI: 10.1063/1.464913

24. Perdew J.P., Burke K., Ernzerhof M. Generalized Gradient Approximation Made Simple // Phys. Rev. Lett., 1996, v. 77(18), pp. 3865-3868. DOI: 10.1103/ PhysRevLett.77.3865

25. Atobe J., Koyasu K., Furuse S., Nakajima A. Anion photoelectron spectroscopy of germanium and tin clusters containing a transition- or lanthanidemetal atom; MGen- $(\mathrm{n}=8-20)$ and MSnn- $(\mathrm{n}=15-17)$ $(\mathrm{M}=\mathrm{Sc}-\mathrm{V}, \mathrm{Y}-\mathrm{Nb}$, and Lu-Ta) // Phys. Chem. Chem. Phys., 2012, v. 14(26), p. 9403. DOI: 10.1039/c2cp23247b

26. Gaussian, Inc., Wallingford CT, 2009.

UDC 538.915

DOI: https://doi.org/10.17308/kcmf.2019.21/756

Received 17.04.2019

Accepted 15.05.2019

\title{
THE EFFECT OF GROUP IIIB TRANSITION METALS ON THE FORMATION OF CLOSED-STRUCTURE GERMANIUM CLUSTERS: A COMPUTER-AIDED EXPERIMENT CONDUCTED IN THE FRAMEWORK OF THE DENSITY FUNCTIONAL THEORY
}

\author{
(c) 2019 N. A. Borshch ${ }^{1}$, S. I. Kurganskii ${ }^{2}$ \\ ${ }^{1}$ Voronezh State Technical University \\ 14, Moskovskii pr., 394026 Voronezh, Russian Federation \\ ${ }^{2}$ Voronezh State University \\ 1, Universitetskaya pl., 394018 Voronezh, Russian Federation
}

\begin{abstract}
Purpose. The paper discusses the modelling results of the spatial structure and electronic properties of the clusters $\mathrm{MeGe}_{16}^{-}$and $\mathrm{MeGe}_{20}^{-}(\mathrm{Me}=\mathrm{Sc}, \mathrm{Y}, \mathrm{Lu})$. It considers the possibility of the synthesis of fullerene-like clusters and clusters with other types of closed structures. Comparative calculations were carried out in the framework of the density functional theory using the SDD basis and three different potentials: B3LYP, B3PW91, and PBEPBE. The authors analysed the influence of the chosen potential on the modelling results of the spatial structure of clusters and their electronic spectra. The validity of theoretical methods was assessed by comparing the calculated electronic spectra with the experimental results on photoelectron spectroscopy.

Results The main isomer of the clusters $\mathrm{MeGe}_{16}^{-}$is the Frank-Kasper polyhedron for all of the three encapsulated metal atoms. For the cluster $\mathrm{ScGe}_{20}^{-}$, an isomer with a prismatic structure is detected experimentally. Endohedral structures have noticeably smaller average binding energies, yet, since their electronic spectra are in good agreement with the experimental spectra (although worse than the spectra of a prismatic isomer), their existence is likely. The main isomers of the $\mathrm{YGe}_{20}^{-}$and $\mathrm{LuGe}_{20}^{-}$clusters have endohedral structures, which can be observed with equal probability in the experiment, since their total spectrum ideally agrees with the experimental one.
\end{abstract}

$\bowtie$ Borshch Nadezda A., borsch-nadya@yandex.ru 
Differences in the structure of the main isomers of the clusters $\mathrm{MeGe}_{20}^{-}$are associated with the size of the scandium, yttrium, and lutetium atoms. The scandium atom has a noticeably smaller atomic radius (1.63 $\AA$ ) than the $\mathrm{Y}$ and Lu atoms (1.78 and $1.72 \AA$ respectively); therefore, with a large number of germanium atoms in the cluster $\mathrm{ScGe}_{20}^{-}$the scandium atom cannot stabilize a closed germanium lattice, and the formation of endohedral clusters becomes almost impossible.

Conclusions. For the clusters $\mathrm{MeGe}_{16}^{-}$, calculations in all three potentials gave identical results. For the clusters $\mathrm{MeGe}_{20}^{-}$, the calculation in the potential B3LYP detected a shift in the highest average bond energies toward fullerene-like structures. The Frank-Kasper structures were the least stable in this calculation. The calculation using the PBEPBE potential showed almost the same binding energies of the Frank-Kasper structures and the fullerene-like structures. The B3PW91-calculation demonstrated a small energy difference between these two types of structures.

Keywords: atomic clusters, electronic structure, fullerenes, density functional theory, photoelectron spectra.

\section{ACKNOWLEDGMENTS}

Расчеты проводились в программе Gaussian09 [26], для визуализации структур использована программа GausView [26]. Для расчетов использованы ресурсы Суперкомпьютерного центра ВГУ.

\section{CONFLICT OF INTEREST}

The authors declare the absence of obvious and potential conflicts of interest related to the publication of this article.

\section{REFERENCES}

1. Kroto H. W., Heath J. R., O’Brien S. C., Curl R. F., Smalley R. E. C60: Buckminsterfullerene. Nature, 1985, v. 318, pp. 162-163. DOI: 10.1038/318162a0

2. Hiura H., Miyazaki T., Kanayama T. Formation of Metal-Encapsulating Si Cage Clusters. Phys. Rev. Lett., 2001, v. 86, p. 1733. DOI: 10.1103/PhysRevLett.86.1733

3. Wang J., Han J. Geometries, stabilities, and electronic properties of different-sized ZrSin $(n=1-16)$ clusters: A density-functional investigation. J. Chem. Phys., 2005, v. 123(6), pp. 064306-064321. DOI: 10.1063/1.1998887

4. Guo L.-J., Liu X., Zhao G.-F. Computational investigation of TiSin $(n=2-15)$ clusters by the densityfunctional theory. J. Chem. Phys., 2007, v. 126(23), pp. 234704-234710. DOI: 10.1063/1.2743412

5. Li J., Wang G., Yao C., Mu Y., Wan J., Han M. Structures and magnetic properties of SinMn $(n=1-15)$ clusters. J. Chem. Phys., 2009, v. 130(16), pp. 164514164522. DOI: $10.1063 / 1.3123805$

6. Borshch N. A., Berestnev K. S., Pereslavtseva N. S., Kurganskii S. I. Geometric structure and electron spectrum of YSi $n-$ clusters $(\mathrm{n}=6-17)$ Physics of the Solid State, 2014, v. 56(6), pp. 1276-1281. DOI: $10.1134 / \mathrm{S} 1063783414060080$

7. Borshch N., Kurganskii S. Geometric structure, electron-energy spectrum, and growth of anionic scandium-silicon clusters ScSin- $(\mathrm{n}=6-20)$. J. Appl. Phys., 2014, v. 116(12), pp. 124302-1 - 124302-8. DOI: $10.1063 / 1.4896528$

8. Borshch N. A., Pereslavtseva N. S., Kurganskii S. I. Spatial structure and electronic spectrum of TiSi $\mathrm{n}$ - Clusters ( $\mathrm{n}=6-18)$. Russian Journal of Physical Chemistry A, 2014. v. 88(10), pp. 1712-1718. DOI: 10.1134/S0036024414100070

9. Borshch N. A., Pereslavtseva N. S., Kurganskii S. I. Spatial and electronic structures of the germanium-tantalum clusters TaGe $\mathrm{n}-(\mathrm{n}=8-17)$. Physics of the Solid State, 2014, vol. 56(11), pp. 23362342. DOI: $10.1134 / \mathrm{S} 1063783414110055$

10. Huang X., Yang J. Probing structure, thermochemistry, electron affinity, and magnetic moment of thulium-doped silicon clusters TmSi n $(n=3-10)$ and their anions with density functional theory. J. Mol. Model., 2018, v. 24(1), p. 29. DOI: 10.1007/s00894-0173566-7

11. Zhang, Y., Yang, J., Cheng, L. J. Probing Structure, Thermochemistry, Electron Affinity and Magnetic Moment of Erbium-Doped Silicon Clusters ErSin $(\mathrm{n}=3-10)$ and Their Anions with Density Functional Theory. Clust. Sci., 2018, v. 29(2), pp. 301-311. DOI: 10.1007/s10876-018-1336-Z

12. Ye T., Luo C., Xu B., Zhang S., Song H., Li G. Probing the geometries and electronic properties of charged Zr2Si n q (n = 1-12, q = \pm 1$)$ clusters. Struct. Chem., 2018, v. 29(1), pp. 139-146. DOI: 10.1007/ s11224-017-1011-2

13. Nguyen M.T., Tran Q. T., Tran V.T. A CASSCF/ CASPT2 investigation on electron detachments from ScSi $n$ - (n=4-6) clusters. J. Mol. Model., 2017, v. 23(10), p. 282. DOI: $10.1007 /$ s00894-017-3461-2

14. Liu Y., Jucai Yang J., Cheng L. Structural Stability and Evolution of Scandium-Doped Silicon Clusters: Evolution of Linked to Encapsulated Structures and Its Influence on the Prediction of Electron Affinities for ScSin $(\mathrm{n}=4-16)$ Clusters. Inorg. Chem., 2018, v. 57(20), pp 12934-12940. DOI: 10.1021/acs. inorgchem.8b02159 
15. Kasper J. S., Hagenmuller P., Pouchard M., Cros C. Clathrate Structure of Silicon Na8Si46 and NaxSi136 (x < 11). Science, 1965, v. 150(3704), pp. 1713-1714. DOI: 10.1126/science.150.3704.1713

16. Borshch N. A., Pereslavtseva N. S., Kurganskii S. I.Electron structure and spectral characteristics of Cd-substituted Ge-based clathrates. Semiconductors, 2010, v. 44(8), pp. 987-992. DOI: 10.1134/ S106378261008004X

17. Borshch N. A., Pereslavtseva N. S., Kurganskii S. I. Electronic structure and spectral characteristics of Zn-substituted clathrate silicides. Semiconductors, 2011, v. 45(6), pp. 713-723. DOI: 10.1134/ S1063782611060066

18. Reveles J. U., Khanna S. N. Electronic counting rules for the stability of metal-silicon clusters. Phys. Rev B., 2006, v. 74(3), pp. 035435-1 - 035435-6. DOI: 10.1103/PhysRevB.74.035435

19. Dunning Jr. T. H. and Hay P. J. in Modern Theoretical Chemistry/Ed. by H. F. Schaefer III, v. 3, Plenum, New York, 1977, pp. 1-28.

20. Lee C., Yang W., Parr R. G. Development of the Colle-Salvetti correlation-energy formula into a func- tional of the electron density. Phys. Rev. B., 1998, v. 37(2), pp. 785-789. DOI: 10.1103/PhysRevB.37.785

21. Becke A. D. Density-functional thermochemistry. III. The role of exact exchange.J. Chem. Phys., 1993, v. 98(7), pp. 1372-377. DOI: 10.1063/1.464913

22. Becke A. D. Density functional calculations of molecular bond energies. J. Chem. Phys., 1986, v. 84(8), pp. 4524-4529. DOI: 10.1063/1.450025

23. Becke A. D. Density-functional thermochemistry. III. The role of exact exchange. J. Chem. Phys., 1993, v. 98(7), pp. 5648-5652. DOI: $10.1063 / 1.464913$

24. Perdew J. P., Burke K., Ernzerhof M. Generalized Gradient Approximation Made Simple. Phys. Rev. Lett., 1996, v. 77(18), pp. 3865-3868. DOI: 10.1103/PhysRevLett.77.3865

25. Atobe J., Koyasu K., Furuse S., Nakajima A. Anion photoelectron spectroscopy of germanium and tin clusters containing a transition- or lanthanidemetal atom; MGen- $(\mathrm{n}=8-20)$ and MSnn- $(\mathrm{n}=15-17)$ $(\mathrm{M}=\mathrm{Sc}-\mathrm{V}, \mathrm{Y}-\mathrm{Nb}$, and $\mathrm{Lu}-\mathrm{Ta})$. Phys. Chem. Chem. Phys., 2012, v. 14(26), p. 9403. DOI: 10.1039/c2cp23247b

26. Gaussian, Inc., Wallingford CT, 2009.
Борш Надежда Алексеевна - к. ф.-м. н., доцент, кафедра высшей математики и физикоматематического моделирования, Воронежский государственный технический университет, Воронеж, Российская Федерация; e-mail: borsch-nadya@yandex.ru. ORCID iD 0000-00029932-881X.

Курганский Сергей Иванович - д. ф.-м. н., профессор, кафедра физики твердого тела и наноструктур, Воронежский государственный университет, Воронеж, Российская Федерация; еmail: kurganskii@phys.vsu.ru. ORCID iD 00000002-4202-0953.
Borshch Nadezda A. - Cand. Sci. (Phys.-Math.), Higher Mathematics and Physical and Mathematical Modelling Department, Voronezh State Technical University, Voronezh, Russian Federation; e-mail: borsch-nadya@yandex.ru. ORCID iD 0000-00029932-881X.

Kurganskii Sergey I. - Dr. Sci. (Phys.-Math.), Professor, Solid State Physic and Nanostructures Department, Voronezh State University, ; Voronezh, Russian Federation; e-mail: kurganskii@phys.vsu. ru. ORCID iD 0000-0002-4202-0953. 